\title{
Association between PD-L1 Expression and the Prognosis and Clinicopathologic Features of Renal Cell Carcinoma: A Systematic Review and Meta-Analysis
}

\author{
Maolei Shen ${ }^{\mathrm{a}}$ Guang Chen ${ }^{\mathrm{b}}$ Qiang Xie ${ }^{\mathrm{c}} \mathrm{Xin} \mathrm{Li}^{\mathrm{a}} \mathrm{Hao} \mathrm{Xu}^{\mathrm{d}}$ Hui Wang ${ }^{\mathrm{a}}$ \\ Shankun Zhao ${ }^{a}$ \\ aDepartment of Urology, Taizhou Central Hospital (Taizhou University Hospital), Taizhou, China; \\ ${ }^{\mathrm{b}}$ Department of Pharmacology, School of Medicine, Taizhou University, Taizhou, China; ' $D e p a r t m e n t ~ o f$ \\ Reproduction, Southern Medical University Affiliate Dongguan People's Hospital, Dongguan, China; \\ ${ }^{d}$ Health Co., 69235 Army of PLA, Jiang Xin, China
}

\section{Keywords}

Renal cell carcinoma $\cdot$ Cell death-ligand 1 - Prognosis .

Clinicopathologic features

\begin{abstract}
The expression of programmed cell death-ligand 1 (PD-L1) and its correlation with the prognosis and clinicopathologic features of renal cell carcinoma (RCC) remain controversial to date. Concerning this issue, we had conducted a metaanalysis of relevant studies searched in the Web of Science, PubMed, EMBASE, and Cochrane Library databases. The Newcastle-Ottawa quality assessment scale was applied to assess the quality of the included studies. The hazard ratio (HR) and its corresponding 95\% confidence intervals (Cls) were collected by Stata 12.0 and used for the results of overall survival (OS) and disease-free survival (DFS). A total of 1,644 patients in 8 studies were included in this meta-analysis. Results showed that PD-L1 expression significantly correlated with OS $(\mathrm{HR}=1.98,95 \% \mathrm{Cl}: 1.22-3.22, Z=2.77, p=$ 0.006 ) and DFS (HR $=3.70,95 \% \mathrm{Cl}: 2.07-6.62, Z=4.40, p=$ 0.0001 ) in ccRCC. Subgroup analysis indicated that PD-L1 ex-
\end{abstract}

karger@karger.com

(c) 2020 S. Karger AG, Basel

www.karger.com/uin

Karger! pression significantly correlated with the lymph-gland transfer ratio $(\mathrm{HR}=2.45,95 \% \mathrm{Cl}: 1.02-5.92, Z=1.99, p=0.05)$ and tumor necrosis ( $\mathrm{HR}=6.05,95 \% \mathrm{Cl}: 3.78-9.67, Z=7.51, p<$ 0.00001 ). This meta-analysis suggests that PD-L1 expression is a valuable prognostic tool for patients with ccRCC. Subgroup analyses demonstrated that it was helpful for screening patients with RCC who need anti-PD-1/PD-L1 treatment and support them to benefit from such immune-targeted therapy.

(c) 2020 S. Karger AG, Basel

\section{Introduction}

According to the GLOBOCAN 2018, kidney cancer is the 16th frequently diagnosed cancer and the third most common genitourinary malignancy among both sexes [1]. It was estimated that there would be a total of 403,262 newly occurring kidney cancer (2.2\% of all cancers) and

Maolei Shen, Guang Chen, and Qiang Xie have contributed equally to this work. 
175,098 kidney cancer deaths ( $1.8 \%$ of all tumor-related deaths) worldwide in 2018, with an increasing incidence [1]. Renal cell carcinoma (RCC) is the commonest solid lesion within the kidney, accounting for nearly $90 \%$ of all kidney malignancies [2]. The most common subtype of kidney cancer is the clear-cell RCC (ccRCC) which accounts for most of the cancer-related deaths. There is a 1.5:1 predominance in males over females. The peak incidence of RCC presents at 60-70 years of age [3]. Etiology includes genetic characteristics and lifestyle factors (i.e., smoking, obesity, and hypertension) [4]. It was reported that up to $30 \%$ of patients with RCC have advanced disease at initial diagnosis, with a 5 -year survival rate of $11 \%$ [5]. Surgical treatment was the first option for the early stage of RCC. However, patients with advanced RCC usually have a poor prognosis because RCC is highly resistant to chemotherapy.

Immune checkpoint inhibitors have shown efficacy in treating multiple solid tumors, including RCC. Since the introduction of vascular endothelial growth factor (VEGF) pathway inhibitors (i.e., sunitinib and sorafenib) in 2005 , these regimens have effectively improved the perspectives of metastatic RCC (mRCC) patients [6]. However, it was reported that the median overall survival (OS) for mRCC patients remained only 12.5 months after first-line targeted therapy [7]. Inspiringly, the development of immune checkpoint inhibitors that block programmed cell death 1 receptor (PD-1) and PD ligand 1 (PD-L1) interaction was proved to conduct very durable responses, reduce tumor volume, and even prolong the OS of patients with RCC [8]. The application of inhibitors of PD-1 and its ligand PD-L1 was considered as the treatment landscape in advanced RCC.

PD-L1 (encoded by the CD274 gene), also known as B7-H1 or CD274, is a ligand for PD-1. PD-L1 mainly expressed on the surface of most tumor cells and immune cells, that is, macrophage, $\mathrm{CD} 4^{+}$cells, $\mathrm{CD} 8^{+} \mathrm{T}$ cells, and adjustment $\mathrm{T}$ cells $[9,10]$. Current evidence proved that multiple cancers expressed high levels of PD-L1. The cancer cells can evade T-cell immunity by exploiting PD-L1/ PD-1 signaling [11]. Through the combination with its corresponding receptor, PD-L1 can dramatically inhibit the excitation, proliferation, and secretion of the relevant cell factor of T cells, thus impeding the immune response, inducing T-cell failure and even causing apoptosis [1215]. Cancer cells, however, can highly express PD-L1 to avoid immune monitoring and thus escape the immune system, which complicates treatment and further leads to poor prognosis [12]. Therefore, the expression of PD-L1 in cancer may serve as a biomarker for predicting and judging the therapeutic effect of immune checkpoint blockade. However, the effect of PD-L1 expression on the prognosis of patients with RCC remains controversial to date. Relevant studies showed that a higher level of PD-L1 expression in RCC associated with large tumor size, high tumor node metastasis (TNM) stage, poor nuclear grade, and increased risk of death $[16,17]$. Inversely, it was reported that RCC patients with low expression of PD-L1 mRNA level have higher tumor staging, tumor grading, and metastatic level than those with high PD-L1 mRNA expression [18]. In this regard, a higher PD-L1 mRNA level in RCC seemed to associate with a favorable outcome in these patients [18].

Presently, results from different studies remain conflicted and inconclusive on the prognostic significance of PD-L1 in patients with RCC, especially RCC. Though most of the relevant researches were inclined to identify PD-L1 as a positive biomarker on RCC, the negative role of PD-L1 should not be overlooked. The evidence for this issue is still controversial among studies, and the comprehensive information is limited to date. Therefore, we would like to conduct a systematic review and meta-analysis in an attempt to investigate the association between PD-L1 expression and the clinicopathologic features of RCC and further explore the prognostic effect of PD-L1 in RCC.

\section{Methods}

The protocol of the present systematic review and meta-analysis was following the Preferred Reporting Items for Systematic Reviews and Meta-Analyses (PRISMA) guidelines. The PRISMA checklist was shown in online suppl. Table 1; for all online suppl. material, see www.karger.com/doi/10.1159/000506296.

Data Sources and Searches

Two authors independently searched relevant studies from the Web of Science, PubMed, EMBASE, and Cochrane Library literature databases. The retrieval time ended on May 15, 2017. The systematic searching was restricted to the English language and human subjects. The following search retrieval keywords were employed in PubMed: "renal cell carcinoma," "RCC," "kidney cancer," "programmed cell death-ligand 1," "PD-L1," "B7-H1," and “CD274."

\section{Inclusion and Exclusion Criteria}

Two authors independently screened the literatures according to the following criteria: (1) studies including patients who were diagnosed with primary RCC after cytology or pathology; (2) studies that used the immunohistochemical method to inspect PD-L1 protein expression in the RCC tissues; (3) studies that focused on PD-L1 expression and patient prognosis, that is, overall survival (OS) and disease-free survival (DFS), and/or the correlation between clinicopathologic features; (4) studies that provided sufficient information to evaluate the hazard ratio (HR); and (5) articles 


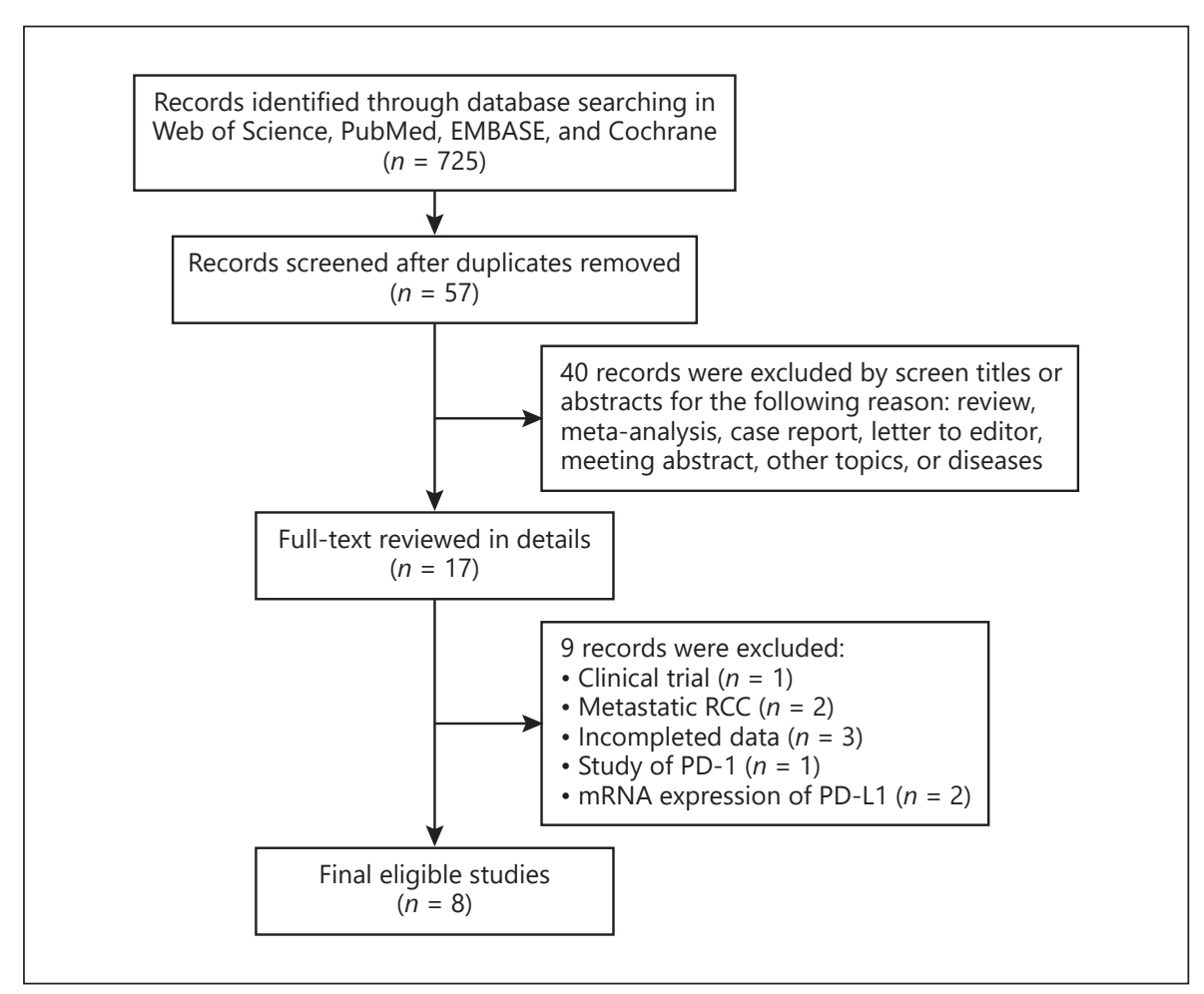

Fig. 1. Flow chart of study selection.

that were published in English. For repeatedly published studies, we only chose the latest literature or the literature with the largest sample size. The articles were excluded based on the following reasons: (1) without the normal control group data; (2) review articles, editorials, comments, letters, and case reports, etc.; (3) duplicated data; and (4) animal experiment.

\section{Data Extraction and Literature}

A standardized data collection form was employed to extract the following information by 2 authors independently: the first authors' name, publication year, country of origin, sample size, immunohistochemistry assessment method, antibody, PD-L1 positive optimum threshold value, general clinicopathological data, OS, DFS, and HR and its 95\% confidence intervals (CIs). In case no direct $\mathrm{HR}$ or $95 \%$ CI was provided in the publications or could not be calculated through the existing data, we tried to contact the corresponding author to obtain the relevant data. If no response was received from the author, data were extracted from the survival curve. In case of disagreements during data extraction, a third author would participate in the discussion.

\section{Quality Assessment}

The Newcastle-Ottawa quality (NOS) assessment scale was used to evaluate the quality of the included studies by the 2 authors independently. The full mark of the scale was 9. Scores with $0-3$, 4-6, and 7-9 were regarded as low quality, moderate quality, and high quality, respectively.

\section{Statistical Analyses}

Data analyses were conducted through the Review Manager 5.3 (The Cochrane Collaboration, Oxford, England) and Stata
12.0 statistical software (Stata Corp LP, College Station, TX, USA). The correlation between PD-L1 expression level and the general clinical data was evaluated by the odds ratio and its $95 \%$ CI. The correlation of PD-L1 expression with OS and DFS was evaluated by $\mathrm{HR}$ with the corresponding 95\% CI. HR and 95\% CI were generated and extracted by the Engauge Digitizer version 4.1 software from the survival curve. The assessment of heterogeneity among the included studies was presented by the $\chi^{2}$ and Higgins $I^{2}$. Substantial heterogeneity has existed when the $I^{2}$ was over $50 \%$. The fixed-effects model was applied for the combined analysis when no significant homogeneity among each of the studies was found $\left(\chi^{2} p>0.01\right.$ or $\left.I^{2}<50 \%\right)$. Otherwise, the random-effects model was applied. Begg's rank correlation test and Egger's regression asymmetry test were adopted to test and evaluate the potential publication bias. $p<0.05$ was considered to reach statistical significance.

\section{Results}

\section{Literature Retrieval Result}

Initially, a total of 874 studies were identified in the present meta-analysis, of which 149 were excluded for duplication. After overviewing the subjects and abstracts, 668 irrelevant studies were excluded, and then 40 more studies were further eliminated for reasons (i.e., reviews, meta-analysis, case reports, letter to the editor, meeting abstracts, and other topics or diseases). The 17 remaining 


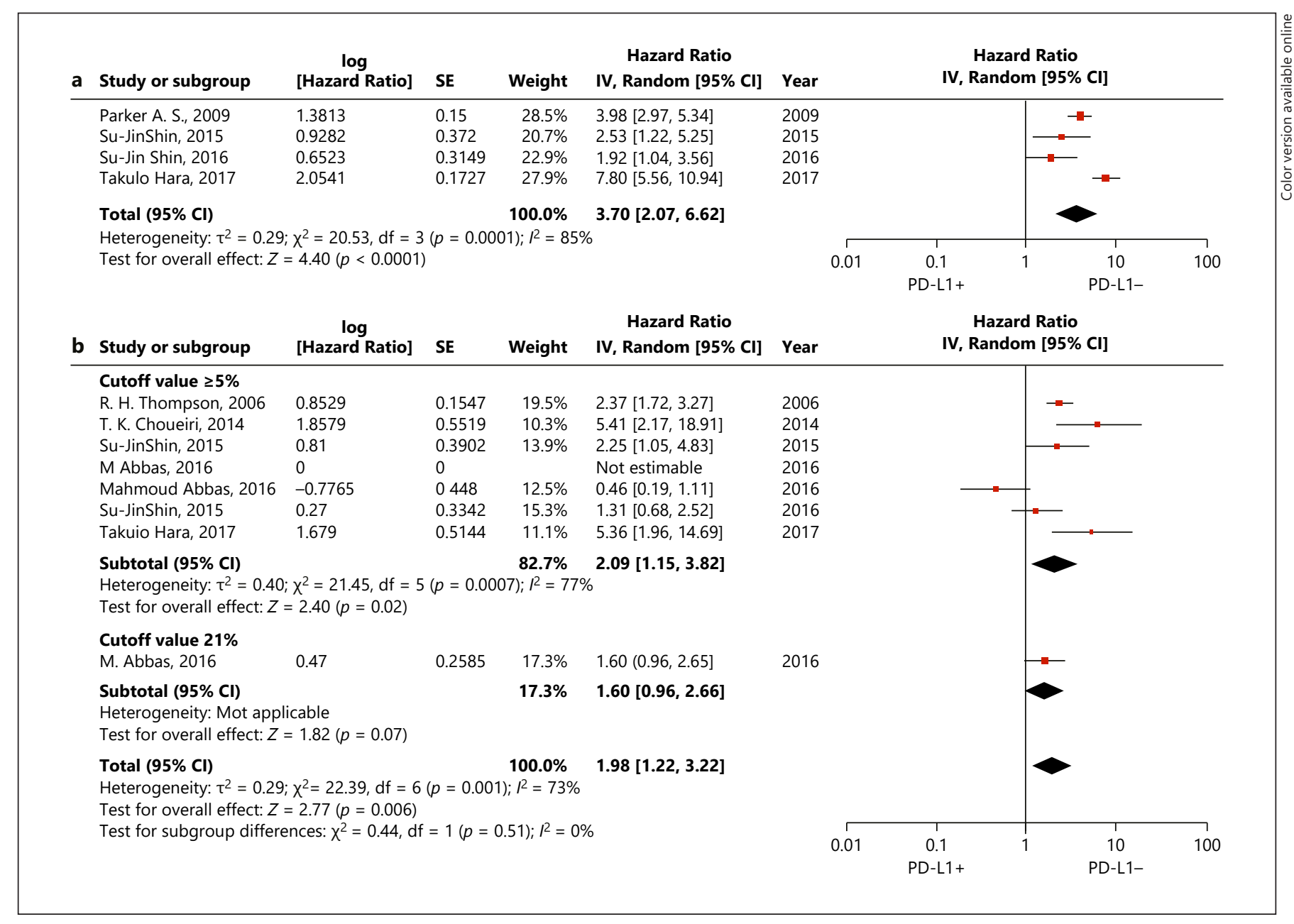

Fig. 2. Forest plot describing the association between PD-L1 expression and OS and DFS of patients with kidney cancer: DFS (a) and OS (b). PD-L1, programmed cell death-ligand 1; OS, overall survival; DFS, disease-free survival.

studies were evaluated for their full texts, leaving 8 eligible studies for this meta-analysis. The literature screening process chart is shown in Figure 1.

The detailed characteristics included in the studies are summarized in online suppl. Table 1 . A total of 1,644 patients were included in the 8 studies [19-26] for the metaanalysis. The 8 included studies were published between 2006 and 2016. There were 3 studies conducted in the USA, 2 in Germany, 2 in Korea, and 1 in Japan. The histologic type of kidney cancer was either "clear cell" or "non-clear cells". Among the 8 eligible studies, 6 studies reported the stage of RCC was I-IV. All of the included studies were employed the percentage of positive cells to evaluate the expression of PD-L1. Among the 8 included studies, all of them were judged as high-quality methodology.

\section{Correlation of PD-L1 Expression with the DFS of Patients with RCC}

Four studies analyzed the relationship of PD-L1 expression to the DFS of patients with renal carcinoma, and significant heterogeneity was found in each study $\left(I^{2}=\right.$ $85 \%, p=0.0001)$. Therefore, the random-effects model was used for analysis. Results indicated that patients with high PD-L1 expression had poor DFS (HR $=3.70$, CI: 2.07-6.62, $Z=4.40, p=0.0001$ ) (Fig. 2a).

\section{Correlation of PD-L1 Expression with the OS of Patients with RCC}

A total of 1,014 patients with RCC in 7 studies were evaluated to determine the correlation between PD-L1 expression and the OS. Significant heterogeneity was found in each study $\left(I^{2}=73 \%, p=0.001\right)$. Therefore, the 


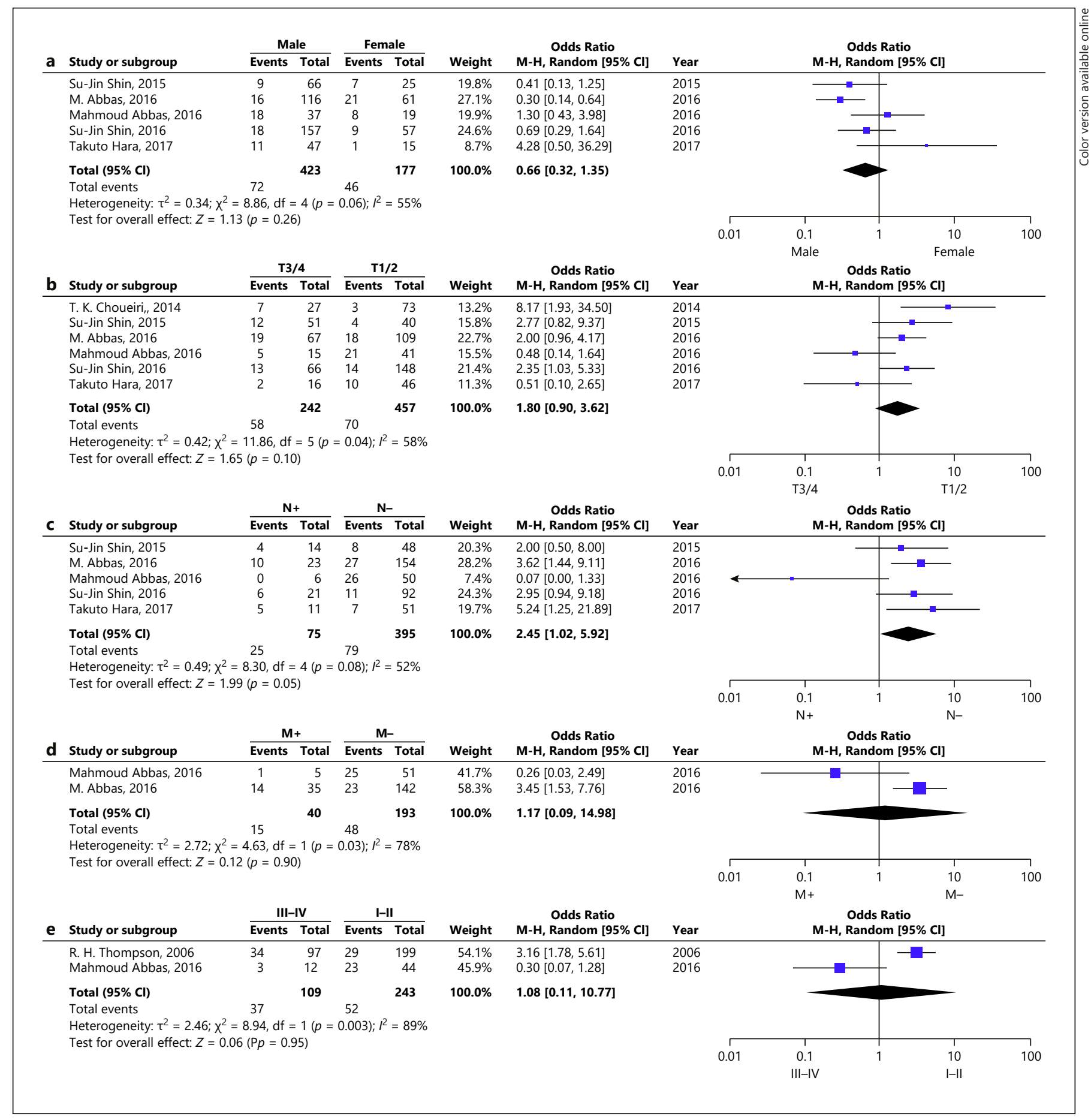

Fig. 3. Forest plots for the association between PD-L1 expression and clinicopathological features: gender (a), depth of invasion (b), lymph node (c), distant metastasis (d), TNM (e), nuclear grade (f), and coagulative tumor necrosis (g). PD-L1, programmed cell death-ligand 1; OS, overall survival; DFS, disease-free survival; TNM, tumor node metastasis.

(Figure continued on next page.) 


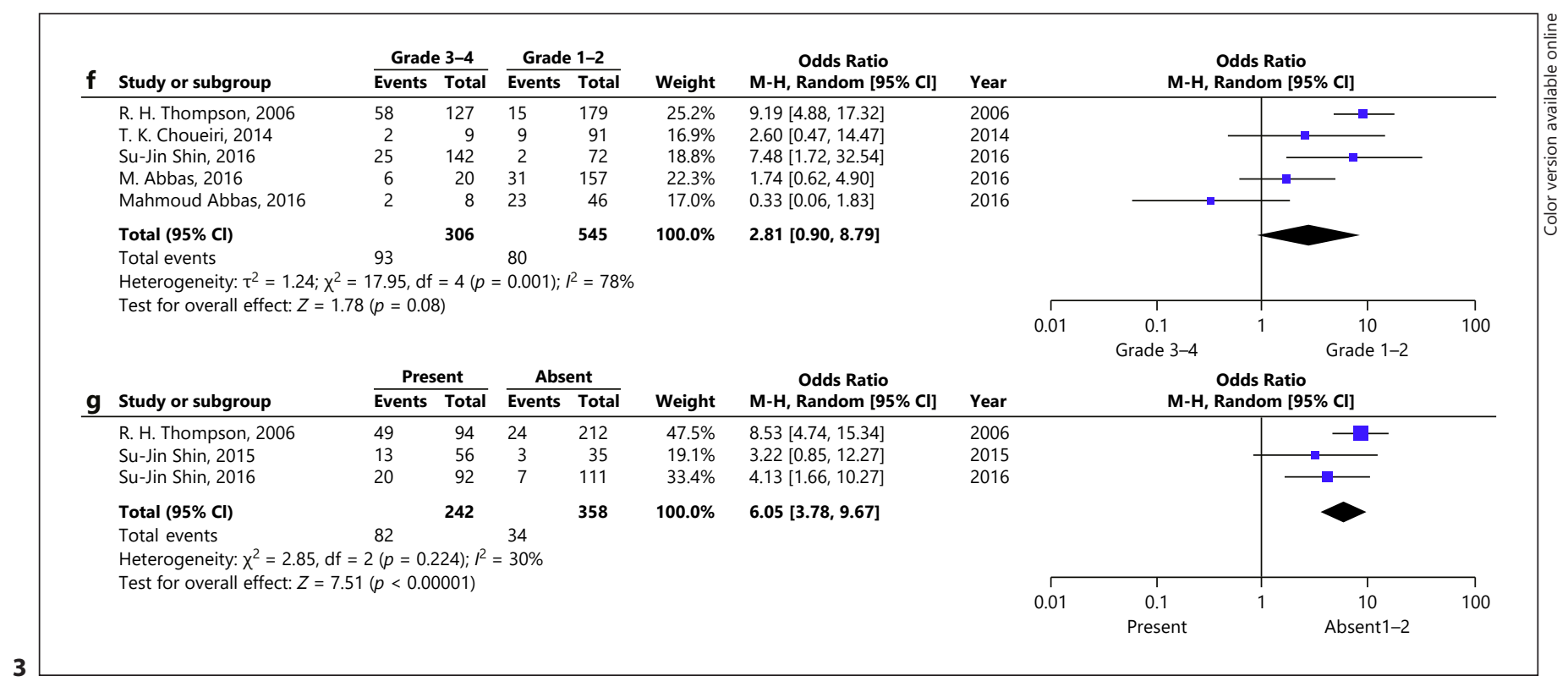

random-effects model was used for the combined analysis. The synthetic results showed that high PD-L1 expression indicated poor prognosis of the patients $(\mathrm{HR}=1.98$, $95 \%$ CI: $1.22-3.22, Z=2.77, p=0.006)$. Subgroup analyses were launched according to the positive optimum threshold value due to the oversize of the overall heterogeneity. The analysis showed that the OS of the RCC patients with high PD-L1 expression was poor when the positive optimum threshold value of PD-L1 was $5 \%$ ( $\mathrm{HR}=2.09,95 \%$ CI: $1.15-3.82, Z=2.40, p=0.02)$ or $1 \%(\mathrm{HR}=1.60,95 \%$ CI: $0.96-2.66, Z=1.82, p=0.07$ ) (Fig. 2b).

Correlation of PD-L1 Expression with the

Clinicopathologic Features of RCC

High PD-L1 expression correlated with high transfer ratio of the lymph gland (HR $=2.45,95 \% \mathrm{CI}: 1.02-5.92$, $Z=1.99, p=0.05)$ and tumor necrosis (HR $=6.05,95 \%$ CI: 3.78-9.67, $Z=7.51, p<0.00001$ ) but not with gender $(\mathrm{HR}=0.66,95 \% \mathrm{CI}: 0.32-1.35, Z=1.13, p=0.26)$, depth of tumor invasion ( $\mathrm{HR}=1.80,95 \% \mathrm{CI}$ : $0.90-3.62, Z=$ $1.65, p=0.10)$, distant metastasis ( $\mathrm{HR}=1.17,95 \% \mathrm{CI}$ : $0.09-14.98, Z=0.12, p=0.90)$, TNM staging $(\mathrm{HR}=1.08$, 95\% CI: $0.11-10.77, Z=0.06, p=0.95)$, and nuclear grading $(\mathrm{HR}=2.81,95 \% \mathrm{CI}: 0.90-8.79, Z=1.78, p=0.08)$ (Fig. 3).

\section{Publication Bias and Sensitivity Analysis}

Based on the publication bias risk assessment and sensitivity analysis by visual inspection, the funnel plot of both DFS and OS was symmetric. The result of Egger's linear regression method (DFS: $p=0.383$; OS: $p=0.971$ ) and Begg's rank correlation test (DFS: $p=0.734$; OS: $p=$ 0.548 ) showed no publication bias in both DFS and OS among the included studies. The unidirectional method was eliminated, but no obvious change in the effect size was observed. This finding indicated that the meta-analysis model was comparatively stable and highly reliable (Fig. 4).

\section{Discussion}

Immunologically, $\mathrm{T}$ cells can selectively recognize and eliminate pathogens as well as the abnormal cells (cancer cells). PD-L1, a coinhibitory immune checkpoint protein which is encoded by the CD274 gene, plays a pivotal role to maintain an intricate regulation of T-cell activities, participating in keeping the homeostasis in the body. Based on a mountain of clinical studies, it was suggested that approximately $30 \%$ malignant tumor cells, including RCC, prostate cancer, testicular cancer, non-small cell lung cancer, melanoma, gastric cancer, breast cancer, glioma, and ovarian cancer, aberrantly express PD-L1 and closely associate with the prognosis of the patients [2732]. The expression of PD-L1 is an immune escape mechanism of the tumor cells. Overexpression of PD-L1 contributes to inhibit the tumor-specific T cell-mediated immunity, that is, inducing $\mathrm{T}$-cell apoptosis and impairing 


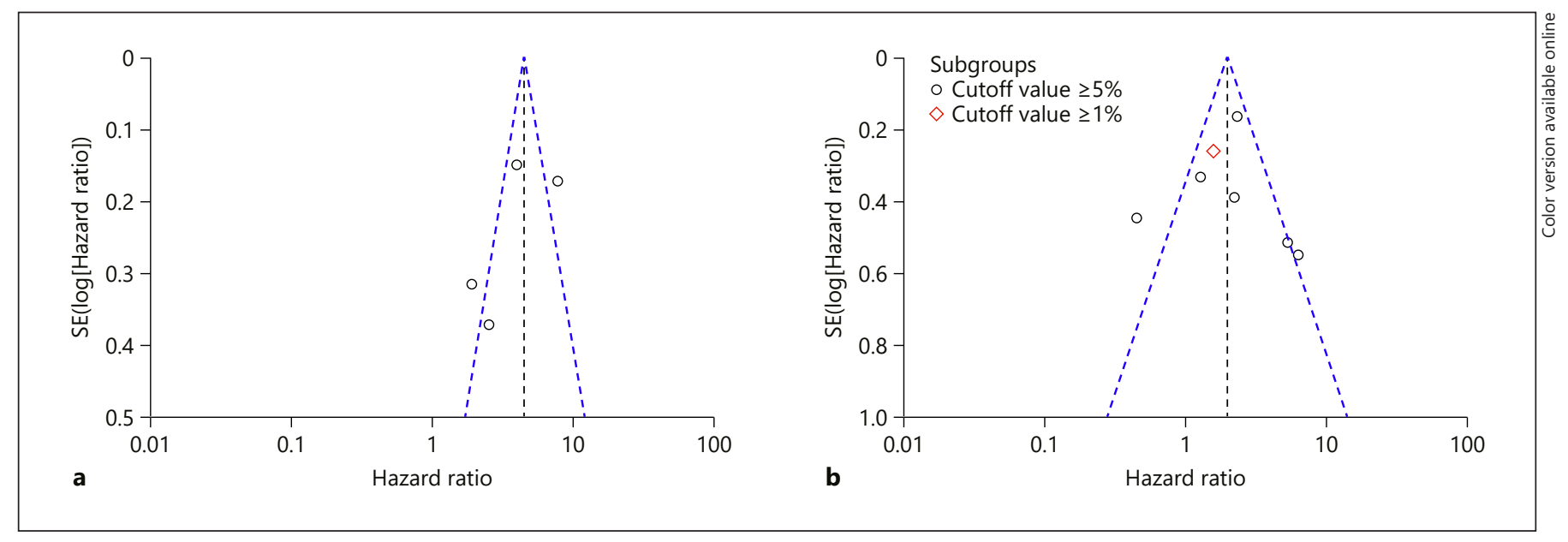

Fig. 4. Funnel blot was designed to visualize a potential publication bias for PD-L1 expression and OS (a) and DFS (b). PD-L1, programmed cell death-ligand 1; OS, overall survival; DFS, disease-free survival.

cytokine production. The combined effects from PD-L1 help the cancer cells to escape the host immune system [33]. Based on this evidence, disrupting the combination of PD-1/PD-L1 may be an effective measure to recover the immune function of T lymphocytes of tumor specificity [34]. In a phase Ia study, the researchers found that atezolizumab, a humanized PD-L1 antibody, has promising antitumor activity in patients with metastatic RCC and has been identified to be a potential predictive and pharmacodynamic biomarker [35]. In the more recent trial of JAVELIN Renal 101, the results showed that in terms of progression-free survival, a PD-L1 inhibitor (avelumab) combined with a multikinase inhibitor (axitinib) was superior to sunitinib as a first-line treatment [36]. These studies have shown that PD-L1 expression can be served as a prognostic tool of tumor progression. Up to now, however, the correlation between the expression level of PD-L1 with the prognosis of RCC remains controversial.

In this synthesized meta-analysis, we have evaluated the correlation of PD-L1 expression with the prognosis of RCC. The results showed that the high expression of PDL1 could reduce the OS and DFS of patients with RCC. Based on pooled HR, our meta-analysis indicated a 1.98to 3.70-fold increased risk of deaths among RCC subjects with high level of PD-L1 compared with those negative/ low expression of PD-L1 (OS: HR $=1.98,95 \%$ CI: $1.22-$ $3.22, Z=2.77, p=0.006$; DFS: $\mathrm{HR}=3.70$, CI: 2.07-6.62, $Z=4.40, p=0.0001)$. Our study was consistent with a previous meta-analysis which also has discovered that the upregulation of $\mathrm{PD}-\mathrm{L} 1$ correlated with poor prognosis of
RCC [37]. However, this meta-analysis included both the ccRCC and non-ccRCC, which might bring the selection bias when synthesized the overall HR. Our study was also in line with a more previous meta-analysis which demonstrated that positive (or higher) PD-L1 expression level was a negative predictor for the cancer-specific survival in RCC patients. This study also concluded that PD-L1 could serve as a key biomarker in the worse prognosis and adverse clinicopathologic features of RCC. However, all data used in this meta-analysis originated from the USA, which could cause a publication bias as substantial heterogeneity across included studies was detected $\left(I^{2}=\right.$ $84.9 \%, p<0.001)$. In addition, the sample size in each of the included study was small; thus, the evidence for the correlation between PD-L1 and DFS was relatively weak. In the present meta-analysis, however, we selected studies strictly in accordance with the inclusion criteria, and all the eligible studies were confirmed with high methodological quality, which made the conclusion more convinced and more suitable for the clinical practice.

Results from the current meta-analysis indicated that PD-L1 overexpression correlated with poor prognosis of RCC. Moreover, we also conducted a subgroup analysis due to the different positive optimum threshold values. The combined results showed that the OS of the RCC patients with high expression of PD-L1 was poor regardless of whether the positive optimum threshold value was $5 \%$ or $1 \%$. The poor prognosis of RCC resulting from PD-L1 overexpression can be elucidated by the following explanation. First, the PD-1/PD-L1 pathway plays a negative regulatory effect in the immunoreaction, which could as- 
sist the tumor cells to escape from the monitoring of the immune system by promoting the inhibition of lymph cells by T-cell apoptosis and proliferation [38]. Furthermore, high expression of tumor-infiltrating lymphocytes indicates a good prognosis, while the tumor-infiltrating lymphocyte expression in tissues overexpressing PD-L1 is low [39]. These conditions may help tumor cells to escape host immunity.

The effective reaction of the drugs, significant survival benefit, and well tolerance of the immune checkpoint paralyzer opened a new channel for the treatment of RCC in fundamental and clinical experiments in the future. The in vitro experiment confirmed that suppressing the $\mathrm{PD} / \mathrm{PD}$-L1 pathway could upregulate $\mathrm{CD} 8^{+} \mathrm{T}$-cell expression and strengthen the endogenous antitumor effect in the body [40]. A recent study has shown that patients with high PD-L1 expression are appropriate to receive targeted treatment [40]. In the present study, the correlation between PD-L1 expression and the clinicopathologic features was analyzed. Results showed that high PD-L1 expression correlated with high lymph-gland transfer ratio and tumor necrosis. Thus, patients with high PD-L1 expression might greatly benefit from PD-L1-targeted treatment. Our study also provided the scientific evidence and theoretical foundation for the clinical application of the PD-1/PD-L1 paralyzer in treating RCC and also indirectly verified that high $\mathrm{PD}-\mathrm{L} 1$ expression is related to the poor prognosis of RCC.

The present meta-analysis had synthesized all the evidence for combining the HR related to the relationship between PD-L1 and the prognosis and clinicopathologic features of RCC. Though our study was comprehensive, our work still had some limitations. First, heterogeneity possibly existed among the results because of the different positive optimum threshold values in the studies. Thus, we conducted a subgroup analysis to further explain the association between PD-L1 and the clinicopathologic features of RCC. Second, some of the studies included in the meta-analysis lacked antibody information. The different antibodies used in the studies probably influenced the accurate evaluation of the PD-L1 positive ratio and the prognosis of RCC. Thus, the same antibody and positive optimum threshold value should be used to obtain accurate results. Third, not all HR in the 95\% CI could be extracted from the studies. As a result, based on the data extracted from the Kaplan-Meier curve, the study was conducted, which could compromise the accuracy of the data. Fourth, this meta-analysis included studies published only in English, resulting in a publication bias. Despite the above limitations, this meta-analysis showed the

correlation between PD-L1 expression and the clinicopathologic characteristics of RCC. The treatment effect of anti-PD-1/PD-L1 could be improved through the convenient descending $a$-stratified method.

In summary, this meta-analysis suggests that PD-L1 expression is a valuable prognostic tool for patients with RCC. In addition, high lymph-gland transfer ratio and tumor necrosis indicated high PD-L1 expression of the patient. This finding could be helpful for screening patients with RCC who need anti-PD-1/PD-L1 treatment and support them to benefit from this immune-targeted therapy.

\section{Statement of Ethics}

For this type of study, formal consent is not required.

\section{Disclosure Statement}

The authors declare that they have no conflict of interest.

\section{Funding Sources}

The authors did not receive any funding.

\section{Author Contributions}

M.L.S., G.C., and S.K.Z. designed this study; X.L. and H.W. searched databases and collected full-text papers; S.X.L. extracted and analyzed data; M.L.S. and S.K.Z. wrote the manuscript; All authors had reviewed the manuscript.

References

Urol Int 2020;104:533-54 DOI: $10.1159 / 000506296$
1 Torre LA, Bray F, Siegel RL, Ferlay J, LortetTieulent J, Jemal A. Global cancer statistics, 2012. CA Cancer J Clin. 2018;65(2):87-108.

2 Ljungberg B, Albiges L, Abu-Ghanem Y, Bensalah K, Dabestani S, Fernández-Pello S, et al European Association of Urology Guidelines on Renal Cell Carcinoma: the 2019 update. Eur Urol. 2019;75(5):799-810.

3 Thorstenson A, Bergman M, Scherman-Plogell $\mathrm{AH}$, Hosseinnia S, Ljungberg B, Adolfsson J, et al. Tumour characteristics and surgical treatment of renal cell carcinoma in Sweden 2005-2010: a population-based study from the National Swedish Kidney Cancer Register. Scand J Urol. 2014;48(3):231-8.

4 Tahbaz R, Schmid M, Merseburger AS. Prevention of kidney cancer incidence and recurrence: lifestyle, medication and nutrition. Curr Opin Urol. 2018;28:62-79. 
5 Fisher R, Gore M, Larkin J. Current and future systemic treatments for renal cell carcinoma. Semin Cancer Biol. 2013;23(1):38-45.

6 Motzer RJ, Rini BI, Bukowski RM, Curti BD, George DJ, Hudes GR, et al. Sunitinib in patients with metastatic renal cell carcinoma. JAMA. 2006;295(21):2516-24.

7 Ko JJ, Xie W, Kroeger N, Lee JL, Rini BI, Knox JJ, et al. The International Metastatic Renal Cell Carcinoma Database Consortium model as a prognostic tool in patients with metastatic renal cell carcinoma previously treated with first-line targeted therapy: a populationbased study. Lancet Oncol. 2015;16(3):293300.

8 Motzer RJ, Escudier B, McDermott DF, George S, Hammers HJ, Srinivas S, et al. Nivolumab versus everolimus in advanced renal-cell carcinoma. N Engl J Med. 2015; 373(19): 1803-13.

9 Zak KM, Kitel R, Przetocka S, Golik P, Guzik $\mathrm{K}$, Musielak B, et al. Structure of the complex of human programmed death $1, \mathrm{PD}-1$, and its ligand PD-L1. Structure. 2015;23(12):2341-8.

10 Miao X, Xu R, Fan B, Chen J, Li X, Mao W, et al. PD-L1 reverses depigmentation in Pmel-1 vitiligo mice by increasing the abundance of Tregs in the skin. Sci Rep. 2018;8(1):1605.

11 Cha JH, Chan LC, Li CW, Hsu JL, Hung MC. Mechanisms controlling PD-L1 expression in cancer. Mol Cell. 2019;76(3):359-70.

12 Wherry EJ, Kurachi M. Molecular and cellular insights into $\mathrm{T}$ cell exhaustion. Nat Rev Immunol. 2015;15(8):486-99.

13 McDermott DF, Atkins MB. PD- 1 as a potential target in cancer therapy. Cancer Med. 2013;2(5):662-73.

$14 \mathrm{Hu}$ Z, Ye L, Xing Y, Hu J, Xi T. Combined SEP and anti-PD-L1 antibody produces a synergistic antitumor effect in B16-F10 melanomabearing mice. Sci Rep. 2018;8(1):217.

15 Caldwell C, Johnson CE, Balaji VN, Balaji GA, Hammer RD, Kannan R. Identification and validation of a PD-L1 binding peptide for determination of PDL1 expression in tumors. Sci Rep. 2017;7(1):13682.

16 Iacovelli R, Nolè F, Verri E, Renne G, Paglino C, Santoni M, et al. Prognostic role of PD-L1 expression in renal cell carcinoma. A systematic review and meta-analysis. Target Oncol. 2016;11(2):143-8.

17 Xu F, Xu L, Wang Q, An G, Feng G, Liu F. Clinicopathological and prognostic value of programmed death ligand-1 (PD-L1) in renal cell carcinoma: a meta-analysis. Int J Clin Exp Med. 2015;8(9):14595-603.

18 Ning XH, Gong YQ, He SM, Li T, Wang JY, Peng SH, et al. Higher programmed cell death 1 ligand 1 (PD-L1) mRNA level in clear cell renal cell carcinomas is associated with a favorable outcome due to the active immune responses in tumor tissues. Oncotarget. 2017; 8(2):3355-63.

19 Shin SJ, Jeon YK, Kim PJ, Cho YM, Koh J, Chung DH, et al. Clinicopathologic analysis of PD-L1 and PD-L2 expression in renal cell aarcinoma: association with oncogenic proteins status. Ann Surg Oncol. 2016;23(2):694702.

20 Parker AS, Leibovich BC, Lohse CM, Sheinin Y, Kuntz SM, Eckel-Passow JE, et al. Development and evaluation of BioScore: a biomarker panel to enhance prognostic algorithms for clear cell renal cell carcinoma. Cancer. 2009; 115(10):2092-103.

21 Abbas M, Steffens S, Bellut M, Becker JU, Grosshennig A, Eggers $\mathrm{H}$, et al. Do programmed death 1 (PD-1) and its ligand (PDL1) play a role in patients with non-clear cell renal cell carcinoma? Med Oncol. 2016;33(6): 59.

22 Hara T, Miyake H, Fujisawa M. Expression pattern of immune checkpoint-associated molecules in radical nephrectomy specimens as a prognosticator in patients with metastatic renal cell carcinoma treated with tyrosine kinase inhibitors. Urol Oncol. 2017;35(6): 363-9.

23 Abbas M, Steffens S, Bellut M, Eggers H, Grosshennig A, Becker JU, et al. Intratumoral expression of programmed death ligand 1 (PD-L1) in patients with clear cell renal cell carcinoma (ccRCC). Med Oncol. 2016;33(7): 80.

24 Choueiri TK, Fay AP, Gray KP, Callea M, Ho $\mathrm{TH}$, Albiges L, et al. PD-L1 expression in nonclear-cell renal cell carcinoma. Ann Oncol. 2014;25(11):2178-84.

25 Shin SJ, Jeon YK, Cho YM, Lee JL, Chung DH, Park JY, et al. The association between PD-L1 expression and the clinical outcomes to vascular endothelial growth factor-targeted therapy in patients with metastatic clear cell renal cell carcinoma. Oncologist. 2015;20(11): 1253-60.

26 Thompson RH, Kuntz SM, Leibovich BC, Dong H, Lohse CM, Webster WS, et al. Tumor B7-H1 is associated with poor prognosis in renal cell carcinoma patients with longterm follow-up. Cancer Res. 2006;66(7): $3381-5$.

27 Soliman H, Khalil F, Antonia S. PD-L1 expression is increased in a subset of basal type breast cancer cells. PloS One. 2014;9(2): e88557.

28 Velcheti V, Schalper KA, Carvajal DE, Anagnostou VK, Syrigos KN, Sznol M, et al. Programmed death ligand-1 expression in nonsmall cell lung cancer. Lab Invest. 2014;94(1): 107-16.

29 Liu Y, Carlsson R, Ambjørn M, Hasan M, Badn W, Darabi A, et al. PD-L1 expression by neurons nearby tumors indicates better prognosis in glioblastoma patients. J Neurosci. 2013;33(35):14231-45.

30 Spranger S, Spaapen RM, Zha Y, Williams J, Meng Y, Ha TT, et al. Up-regulation of PD$\mathrm{L} 1, \mathrm{IDO}$, and $\mathrm{T}$ (regs) in the melanoma tumor microenvironment is driven by $\mathrm{CD} 8(+) \mathrm{T}$ cells. Sci Transl Med. 2013;5(200):200ra116200r.

31 Martin AM, Nirschl TR, Nirschl CJ, Francica BJ, Kochel CM, van Bokhoven A, et al. Paucity of PD-L1 expression in prostate cancer: innate and adaptive immune resistance. Prostate Cancer Prostatic Dis. 2015;18(4): 325-32.

32 Thompson RH, Gillett MD, Cheville JC, Lohse CM, Dong H, Webster WS, et al. Costimulatory $\mathrm{B} 7-\mathrm{H} 1$ in renal cell carcinoma patients: indicator of tumor aggressiveness and potential therapeutic target. Proc Natl Acad Sci U S A. 2004;101(49):17174-9.

33 Iwai $Y$, Ishida $M$, Tanaka $Y$, Okazaki T, Honjo T, Minato N. Involvement of PD-L1 on tumor cells in the escape from host immune system and tumor immunotherapy by PD-L1 blockade. Proc Natl Acad Sci U S A. 2002; 99(19):12293-7.

34 Lee CH, Motzer RJ. Immune checkpoint therapy in renal cell carcinoma. Cancer J. 2016; 22(2):92-5.

35 McDermott DF, Sosman JA, Sznol M, Massard C, Gordon MS, Hamid O, et al. Atezolizumab, an anti-programmed death-ligand 1 antibody, in metastatic renal cell carcinoma: long-term safety, clinical activity, and immune correlates from a phase Ia atudy. J Clin Oncol. 2016;34(8):833-42.

36 Motzer RJ, Penkov K, Haanen J, Rini B, Albiges L, Campbell MT, et al. Avelumab plus axitinib versus sunitinib for advanced renalcell carcinoma. N Engl J Med. 2019;380(12): 1103-15.

37 Weng YM, Peng M, Hu MX, Yao Y, Song QB. Clinical and molecular characteristics associated with the efficacy of PD-1/PD-L1 inhibitors for solid tumors: a meta-analysis. Onco Targets Ther. 2018;11:7529-42.

38 Pardoll DM. The blockade of immune checkpoints in cancer immunotherapy. Nat Rev Cancer. 2012;12(4):252-64.

39 Prado-Garcia H, Romero-Garcia S, AguilarCazares D, Meneses-Flores M, Lopez-Gonzalez JS. Tumor-induced CD8+ T-cell dysfunction in lung cancer patients. Clin Dev Immunol. 2012;2012:741741.

40 Duraiswamy J, Freeman GJ, Coukos G. Therapeutic PD-1 pathway blockade augments with other modalities of immunotherapy Tcell function to prevent immune decline in ovarian cancer. Cancer Res. 2013;73(23): 6900-12. 